\title{
INTERVAL TOPOLOGY IN SUBSETS OF TOTALLY ORDERABLE SPACES ${ }^{1}$ )
}

\author{
BY \\ MARY ELLEN RUDIN
}

A topological space is said to be totally orderable if the points of the space can be totally ordered in such a way that the interval topology induced by this ordering coincides with the given topology. Unfortunately, the property of being a totally orderable space is not hereditary.

Suppose that $R$ is a topological space and that $T$ is a subspace of $R$ and that $R$ is totally orderable. Theorem II of this paper will answer question 1963.4 of Nieuw Archief Voor Wiskunde by giving necessary and sufficient conditions for $T$ to be a totally orderable space. However, the most interesting case is where $R$ is the real line; the solution in this case is quite simple to state and understand and is given in Theorem I. The bibliography lists several papers by I. L. Lynn each of which gives some partial results in the case where $R$ is the real line.

If $\Delta$ is a total ordering of a set $T$, then we will say that $I$ is an interval of $\Delta(T)$ if $I \subset T$ and no element of $T-I$ is between two elements of $I$ in $\Delta$. We will say that $I$ is an open interval of $\Delta(T)$ if there is a last element of $T-I$ preceding every element of $I$ if any element does so, and there is a first element of $T-I$ following every element of $I$ if any element does so. If $T$ is a topological space and $\Delta$ a total ordering of the points of $T$, then we will say that $\Delta(T)$ has interval topology at a point $p$ of $T$ if the open intervals of $\Delta(T)$ form a neighborhood basis for $p$ in $T$. Then $T$ is totally orderable if and only if there is a total ordering of $T$ with interval topology at every point.

I. Consider the following examples of subsets of the real line which are not totally orderable.

EXAMPLE 1. Let $T=(0,1) \cup[2,3]$.

EXAmple 2. Let $T=X \cup X_{1} \cup X_{2} \cdots$, where $X=[0,-1]$ and, for $n$ odd, $X_{n}=[1 / 2 n, 1 / 2 n+1]$, and, for $n$ even, $X_{n}=(1 / 2 n, 1 / 2 n+1)$.

The conditions given in Theorem I prevent precisely the generalized cases of these examples.

I.A. THEOREM I. Suppose that $R$ is the real line and that $T$ is a subspace of $R$. Then the following two conditions are necessary and sufficient for $T$ to be a totally orderable space.

Presented to the Society, August 27, 1963; received by the editors January 20, 1964.

(1) This research was sponsored in part by the National Science Foundation, contract number GP-3857. 
Closures will be taken in $R$. And $Q$ will denote the union of all nontrivial components of $T$ all of whose endpoints belong to the closure of $(\bar{T}-T)$.

Condition 1. If $(T-Q)$ is compact and $(T-Q) \cap \bar{Q}=0$, then either $Q=0$ or $(T-Q)=0$.

Condition 2. If $I$ is an open interval of $R$ and $p$ is an endpoint of $I$ and $\{p\} \cup(I \cap(T-Q))$ is compact and $\{p\}$ is the intersection of the closures of $(I \cap Q)$ and $(I \cap(T-Q))$, then the component of $T$ containing $p$, if any, is trivial.

I.B. The proof of the necessity of the conditions given in Theorem I is rather trivial, so let us dispose of this first.

\section{Lemma 1. Condition 1 is necessary.}

Proof. Suppose that $(T-Q)$ is compact, that $(T-Q) \cap \bar{Q}=0$, that $q \in Q$ and $p \in(T-Q)$, and that $\Delta$ is a total ordering of the points of $T$ with interval topology at every point of $T$. Also suppose that $p \Delta q$. Then, since $(T-Q)$ is compact, there is a $p^{\prime} \in(T-Q)$ such that $p^{\prime} \Delta q$ but there is no point of $(T-Q)$ between $p^{\prime}$ and $q$ in $\Delta$. Since $p^{\prime} \notin \bar{Q}$, there is an open interval I of $\Delta(T)$ containing $p^{\prime}$ but not intersecting $Q$. Let $q^{\prime}$ be the first point of $(T-I)$ following all of the points of $I$ in $\Delta$. By the definition of $p^{\prime}, q^{\prime} \in Q$ and there is no point of $T$ between $q^{\prime}$ and $p^{\prime}$ in $\Delta$. But then, by the definition of $Q, q^{\prime} \notin Q$, which is a contradiction.

\section{LEMMA 2. Condition 2 is necessary.}

Proof. Suppose that $I$ is an open interval of $R$, that $p$ is an endpoint of $I$, that $\{p\} \cup(I \cap(T-Q))$ is compact, that $\{p\}$ is the intersection of the closures of $(I \cap Q)$ and $(I \cap(T-Q))$, and that $t$ is a point distinct from $p$ in the component of $T$ containing $p$. Also suppose that there is a total ordering $\Delta$ of the points of $T$ having interval topology at every point of $T$. Then there is no point of $I \cap T$ between $p$ and $t$ in $\Delta$. Suppose that $t \Delta p$. Since $p$ belongs to the closure of $(I \cap T)$, there is a point $i \in(I \cap T)$ such that $p \Delta x$ for all $x$ between $i$ and $p$ in $I$ and such that $x \in I$ for all $x$ between $i$ and $p$ in $\Delta$. Since $p$ belongs to the closure of $(I \cap Q)$, there is a point $q \in Q$ such that $p \Delta q \Delta i$. And, since $p$ also belongs to the closure of $(I \cap(T-Q))$ and $\{p\} \cup(I \cap(T-Q))$ is compact, there is a point $p^{\prime} \in(I \cap(T-Q))$ such that $p \Delta p^{\prime} \Delta q$ but there is no point of $T-Q$ between $q$ and $p^{\prime}$ in $\Delta$. And by the argument given in Lemma 1 , this is impossible.

I.C. In order to prove the sufficiency of the conditions of Theorem I, we will now make a number of definitions.

Let $R^{\prime}$ denote the compactification of $R$ obtained by adding the points at infinity. Then replace each trivial component of $\left(R^{\prime}-T\right)$ by a simple closed interval. The resulting space $S$ will be compact and connected, $T$ will be a subspace of $S$, 
there will be no trivial components of $(S-T)$, the endpoints of $S$ will not be in $T$, and there will be a total ordering $\Delta$ of $S$ having interval topology at every point of $S$ which preserves the natural order of $T$ in $R$. Because of these nice properties of $S$ we will use $S$ as our basic underlying space rather than $R$. For the rest of our proof of Theorem 1 , unless otherwise specified, closure will be taken in $S$. Observe that $Q$ remains unchanged if we define $Q$ as in Theorem I taking closures in $S$ rather than in $R$. And, if Conditions 1 and 2 are satisfied, they they are satisfied with $R$ replaced by $S$.

Let $P$ be the set of all points $p$ of $S$ for which there is an open interval $I$ of $S$ such that $p$ is an endpoint of $I,\{p\} \cup(I \cap(T-Q))$ is compact, and $\{p\}$ is the intersection of the closures of $(I \cup Q)$ and $(I \cap(T-Q))$.

Observe that Condition 2 may be stated in the following way.

Condition 2'. If $p \in P$, then the component of $T$ containing $p$, if any, is trivial.

Let $H$ be the union of all open intervals $I$ of $S$ such that $I \cap(T-Q)$ is compact and disjoint from $I \cap \bar{Q}$.

Since $S$ is compact, Condition 1 may be stated in the following way.

Condition 1'. If $S \subset H$, then either $T \subset Q$ or $Q=0$.

I.D. We can now describe the special roles of Conditions 1 and 2.

Lemma 3. If $S \subset H$, then Condition 1 is sufficient to insure that $T$ is totally orderable.

Proof. Suppose that $S \subset H$ and that Condition 1, and hence Condition 1', is satisfied. Then either $T \subset Q$ or $Q=0$. If $Q=0$, then, by the definition of $H$, $T$ is compact and, hence, $\Delta(T)$ has interval topology at every point of $T$. And, if $T \subset Q, \Delta(T)$ again has interval topology at every point of $T$.

Lemma 4. If $S \notin H$, then Condition 2 is sufficient to insure that $T$ is totally orderable.

The rest of part I of the paper will be devoted to proving Lemma 4 which will complete the proof of Theorem I; but the proof is long. However, it is notable that this highly specialized local condition plays such a large role in the proof of the theorem.

I.E. We need Lemma 5 in order to prove Lemmas 6 and 7.

Lemma 5. Suppose that Condition 2 holds and that $I$ and $J$ are disjoint open intervals of $\Delta(S)$ contained in $H$ and having a common endpoint $x$ not in $H$. Then, if $J \subset T, J \subset(T-Q)$ and $x$ is not in the closure of $I \cap(T-Q)$.

Proof. First suppose that our hypotheses hold. Since $J \subset T$, either $J \subset Q$ or $J \subset(T-Q)$. Suppose $J \subset Q$. By Condition 2, $x$ is not in the closures of both $(I \cap Q)$ and $(I \cap(T-Q))$. If $x$ is either not in $T$ or not in the closure of $(I \cap(T-Q))$, then there is an open interval $I^{\prime}$ containing $x$ which intersects $T$ 
only in $Q$; hence $I^{\prime} \subset H$; but this contradicts the fact that $x$ is not in $H$. But, if $x$ belongs to $T$ but not to the closure of $(I \cap Q)$, since $J \subset Q$, by the definition of $Q$ there is a point $i \in(I-T)$ in the closure of $(T-Q)$. And, by the definition of $H$, there is, hence, an open subinterval $I^{\prime}$ of $I$ containing $i$ such that $I^{\prime} \cap(T-Q)$ is compact which contradicts the definition of $i$. Hence $J \subset(T-Q)$.

Now suppose that $x$ belongs to the closure of $(I \cap(T-Q))$. Since the components of $(S-T)$ are nontrivial, $x \in T$, and, by Condition $2, x$ does not belong to the closure of $(I \cap Q)$. So there is a point $i$ of $I$ such that the interval $[i, x]$ of $S$ does not intersect $Q$. But, since $I \subset H,[i, x] \cap T$ is compact; hence $((i, x] \cup J) \subset H$ which contradicts the fact that $x$ is not in $H$.

Lemma 6. Suppose that Condition 2 holds, that $X$ is an open interval $(p, q)$ of $S$, that $p$ and $q$ are not in $T$, and that $(X-H)$ is a single point $x$. Then there is a total ordering $\Theta$ of $(X \cap T)$ with interval topology at every point of $(X \cap T)$ such that $\Theta(X \cap T)$ has a last point but no first point.

Proof. Without loss of generality we will assume that $p<q$. Let $I$ and $J$ denote the open intervals $(p, x)$ and $(x, q)$ of $S$. In each of the cases discussed below, let $\Theta(J \cap(T-Q))=\Delta(J \cap(T-Q))$, let $\Theta(I \cap(T-Q))=-\Delta(I \cap(T-Q)$, let $\Theta(I \cap Q)=\Delta(I \cap Q)$, let $\Theta(J \cap Q)=-\Delta(J \cap Q)$, and let

$$
((I \cup J) \cap Q) \Theta(\{x\} \cap T) \Theta((I \cup J) \cap(T-Q)) .
$$

Case 1. Suppose that $x$ is a limit point of a component $J^{\prime}$ of $J \cap T$. Then, by Lemma $5, J^{\prime} \subset(T-Q)$ and $x$ is not in the closure of $(I \cap(T-Q))$. In this case, let $(J \cap Q) \Theta(I \cap Q)$ and $(J \cap(T-Q)) \Theta(I \cap(T-Q))$. The ordering $\Theta$ of $(X \cap T)$ so defined has the desired properties and the case where $x$ is a limit point of a component of $I \cap T$ can be similarly handled.

Case 2. Suppose there is no nontrivial component of $T$ having $x$ in its closure. Then there are monotonic sequences $i_{1}, i_{2}, \cdots$ and $j_{1}, j_{2}, \cdots$ of points of $I-T$ and $J-T$, respectively, approaching $x$. Define $p=i_{0}$ and $q=j_{0}$. Then for each positive integer $n$, let $\left(\left(i_{n}, i_{n-1}\right) \cap Q\right) \Theta\left(\left(j_{n}, j_{n-1}\right) \cap Q\right) \Theta\left(\left(i_{n+1}, i_{n}\right) \cap Q\right)$ and let $\left(\left(i_{n}, i_{n-1}\right) \cap(T-Q)\right) \Theta\left(\left(j_{n}, j_{n-1}\right) \cap(T-Q)\right) \Theta\left(\left(i_{n+1}, i_{n}\right) \cap(T-Q)\right)$. Again $\Theta$ has the desired properties.

I.F. Let $H^{\prime}$ be the union of all open intervals of $S$ containing at most one point not in $H$. Then define $G$ as the collection of all closures of components of $H^{\prime}$. These terms of $G$ will be the basic sets throughout the rest of our proof of Lemma 4. Using Lemmas 6 and 7 we can make a certain construction which allows us to order $T$ fairly well within each element of $G$ if Condition 2 holds. Lemmas $8,9,10,11$, and 12 say how well. Let $G^{\prime}$ denote the set of all terms of $G$ whose interiors are not in $H$.

Lemma 7. Suppose that Condition 2 holds, that $X \in G^{\prime}$, and that $J$ is a component of $X \cap H$. Then $J \notin T$. 
Proof. Suppose the contrary. Since $X \in G^{\prime}$, there is a component $I$ of $X \cap H$ having a common endpoint with $J$ where $I \neq J$. So, by Lemma $5, J \subset(T-Q)$. Hence there is an endpoint $j$ of $J$ not in the closure of $\bar{T}-T$. So there is an open interval $J^{\prime}$ of $S$ containing $j$ such that $\left(\bar{J}^{\prime} \cap T\right)$ is compact. But then $J^{\prime} \subset H$ and $J$ is not a component of $H$.

I.G. We are now ready to describe our construction.

Suppose that $K \in G^{\prime}$ and let $K^{\prime}$ denote the interior of $K$. By Lemma 7, there is a subset $Z$ of $\left(K^{\prime}-T\right)$ containing one and only one point from each component of $K \cap H$. Let $Y$ denote the set of all components of $K-Z$ both of whose endpoints are in $K^{\prime}$. Since $K \in G^{\prime}, Y \neq 0$ and, since each element $X$ of $Y$ satisfies the hypotheses of Lemma 6, we can find, for each element $X$ of $Y$, a total ordering $\Theta$ of $X \cap T$ having interval topology at every point of $X \cap T$ such that $\Theta(X \cap T)$ has a last point but no first point. The elements of $Y$ have a natural order in $\Delta(S)$ and we choose an element $Y_{0}$ of $Y$ so that $Y_{0}$ is the first element of $Y$ if such exists. Let $Y^{*}$ denote the union of the closures of the elements of $Y$. Then there are total orderings $\Lambda$ and $\Lambda^{\prime}$ of $\left(Y^{*} \cap T\right)$ as follows. If $U$ and $V$ belong to $Y$, let $(U \cap T) \Lambda(V \cap T)$ or $(U \cap T) \Lambda^{\prime}(V \cap T)$ if and only if $(U \cap T) \Delta(V \cap T)$. And, if $X \in Y$, let $\Lambda(X \cap T)=\Theta(X \cap T)$ and $\Lambda^{\prime}(X \cap T)=-\Theta(X \cap T)$ if either $X=Y_{0}$ or the number of terms of $Y$ between $X$ and $Y_{0}$ is odd; and let $\Lambda(X \cap T)=-\Theta(X \cap T)$ and $\Lambda^{\prime}(X \cap T)=\Theta(X \cap T)$ if the number of terms of $Y$ between $X$ and $Y_{0}$ is even. Observe that both $\Lambda\left(Y^{*} \cap T\right)$ and $\Lambda^{\prime}\left(Y^{*} \cap T\right)$ have interval topology at every point of $\left(Y^{*} \cap T\right)$ and that, if $Y_{0}$ is the first term of $Y, \Lambda\left(Y^{*} \cap T\right)$ has no first point but $\Lambda^{\prime}\left(Y^{*} \cap T\right)$ has a first point.

If $p$ is an endpoint of a compact interval $K$, then we say that $p \in P_{K}$ provided that there is a component $I$ of $K \cap H$ such that $p$ belongs to the closures of both $(I \cap Q)$ and $(I \cap(T-Q))$.

If $p \Delta q$ and $I$ is the interval $[p, q]$ of $S$ and $\Pi$ is a total ordering of $(I \cap T)$, then we say that $\Pi$ orders $T$ nicely in $I$ if:

(1) $\Pi(I \cap T)$ has interval topology at every point of $(I \cap T)$,

(2) if $p \in T, p$ is the first point of $\Pi(I \cap T)$ and, if $q \in T, q$ is the last point of $\Pi(I \cap T)$,

(3) if $p \notin T$, there is no first point of $\Pi(I \cap T)$, and, if $q \notin T$, there is no last point of $\Pi(I \cap T)$.

I.H. Throughout this section we assume that Condition 2 holds, that $K$ is an element $[p, q]$ of $G^{\prime}$, and that the construction described in $\S I$. G has been made. And we prove a number of lemmas about the possible orderings of $(K \cap T)$.

Lemma 8. Suppose that Condition 2 holds, that $[p, q]$ is an element $K$ of $G^{\prime}$, and that $p \in P_{K}$ but $q \notin P_{K}$. Then there is an $x \in(p, q)$ and a total ordering $\Pi$ of $([x, q] \cap T)$ such that $(p, x] \subset H$ and $\Pi$ orders $T$ nicely in $[x, q]$. 
Assume that $p \Delta q$ and construct $Z, Y, Y^{*}, Y_{0}, \Lambda$, and $\Lambda^{\prime}$ as described in $G$. Let $y$ denote the first point of $Y^{*}$; by the definitions of $p$ and $Z$ there is such a point. Now we will define $x$ and $\Pi$ of our conclusion by considering various cases.

Case 1. Suppose that $q$ is not in the closure of $Y^{*}$. Then there is a component $X$ of $((p, q)-Z)$ having $q$ on its boundary, the number of terms of $Y$ is finite, and, since $q$ is not in $P_{K}, q$ is not in the closure of both $(X \cap(T-Q))$ and $(X \cap Q)$.

In both Cases 1.a and 1.b, let $\Pi(X \cap(T-Q))=\Delta(X \cap(T-Q))$ and $\Pi(X \cap Q)=\Delta(X \cap Q)$.

Case 1.a. Suppose that either $q$ does not belong to $T$ or that $q$ does belong to the closure of $(X \cap Q)$.

If the number of terms of $Y$ is even, let $x=y$, let $\Pi\left(Y^{*} \cap T\right)=\Lambda\left(Y^{*} \cap T\right)$, and let $\left(Y_{0} \cap T\right) \Pi(X \cap(T-Q)) \Pi\left(\left(Y^{*}-Y_{0}\right) \cap T\right) \Pi(X \cap Q) \Pi(\{q\} \cap T)$.

If the number of terms of $Y$ is odd, then select the last point $z$ of $((p, y) \cap(T-Q))$ in $\Delta$, and let $x=z$. Let $\Pi\left(Y^{*} \cap T\right)=\Lambda^{\prime}\left(Y^{*} \cap T\right)$, let $\Pi((z, y) \cap Q)=\Delta((z, y) \cap Q)$ and let

$$
\Pi(X \cap(T-Q)) \Pi\left(Y^{*} \cap T\right) \Pi((z, y) \cap Q) \Pi(X \cap Q) \Pi(\{q\} \cap T) .
$$

Case 1.b. Suppose that $q$ belongs to $T$ but not to the closure of $(X \cap Q)$. Then we can define $x$ as in Case 1.a interchanging even and odd. And, if the number of terms of $Y$ is odd, let $(X \cap Q) \Pi\left(Y^{*} \cap T\right) \Pi(X \cap(T-Q)) \Pi\{q\}$, and if the number of terms is even, let

$$
\Pi(X \cap(T-Q)) \Pi\left(Y_{0} \cap T\right) \Pi((z, y) \cap Q) \Pi(X \cap Q) \Pi\left(\left(Y^{*}-Y_{0}\right) \cap T\right) \Pi\{q\} .
$$

Case 2. Suppose that $q \in \bar{Y}$. Let $x=y$. Since $q \in \bar{Y}, Y$ has infinitely many terms and $q$ is their sequential limit point; so let $\Pi\left(Y^{*} \cap T\right)=\Lambda\left(Y^{*} \cap T\right)$, and, if $q \in T$, let $\left(Y^{*} \cap T\right) \Pi\{q\}$.

Lemma 9. Suppose that Condition 2 holds, that $p \in P_{K}$ and $q \in P_{K}$, that $K \in G$, and that $[p, q]=K$. Then, if $p \Delta q$, there are two points $x$ and $y$ of $(p, q)$ such that $x \Delta y,(p, x] \subset H,[y, q) \subset H$, and there is a total ordering $\Pi$ of $(T \cap[x, y])$ which orders $T$ nicely in $[x, y]$.

Proof. If $(p, q) \subset H$, then let $x$ and $y$ be two points belonging to the same component of $((p, q)-T)$ such that $x \Delta y$. These points trivially have the desired property since $(T \cap[x, y])=0$.

Suppose $(p, q) \notin H$. Then $K \in G^{\prime}$ and we can use the construction described in $\S I . G$ to define $Z, Y, Y^{*}, Y_{0}, \Lambda$, and $\Lambda^{\prime}$. Since there are components of ( $H \cap(p, q)$ ) having $p$ and $q$ on their boundaries, the number of terms of $Y$ is finite and there will be a first point $w$ of $Y^{*}$ and a last point $z$ of $Y^{*}$. Let $x=w$. If the number of terms of $Y$ is even we let $y=z$ and observe that $\Lambda$ orders $T$ nicely in $Y^{*}$ so we can let $\Pi=\Lambda$. If the number of terms of $Y$ is odd, let $y$ be 
the first point of $\Delta([z, q])$ belonging to $(T-Q)$. Then let $\Pi$ be the total ordering of $(T \cap[x, y])$ such that $\Pi\left(Y^{*} \cap T\right)=\Lambda\left(Y^{*} \cap T\right)$ and $\Pi([y, z) \cap Q)=\Delta([y, z) \cap Q)$ and $([y, z) \cap Q) \Pi\left(Y^{*} \cap T\right) \cdot \Pi z$.

Lemma 10. Suppose that Condition 2 holds, that $K \in G$, and that one endpoint of $K$ is not a boundary point of any component of $(K \cap H)$ and the other is not in $P_{K}$. Then there is a total ordering $\Pi$ of $(K \cap T)$ which orders $T$ nicely in $K$.

Proof. Let $p$ be one endpoint of $K$ which is not a boundary point of any component of $(K \cap H)$ and let $q$ be the other endpoint of $K$. Without loss of generality we assume that $q \Delta p$. Since there are infinitely many components of $(K \cap H), K \in G^{\prime}$, and we can use the construction of §I.G to define $Z, Y, Y^{*}$, $Y_{0}, \Lambda$, and $\Lambda^{\prime}$. Observe that $Y$ is infinite and $p \in \bar{Y}^{*}$. Our proof is analogous to that of Lemma 8.

Case 1. Suppose that $q \notin \bar{Y}^{*}$ and let $X$ be the component of $(K-Z)$ containing $q$. In both Cases 1.a and 1.b let $\Pi(X \cap(T-Q))=\Delta(X \cap(T-Q))$ and $\Pi(X \cap Q)=\Delta(X \cap Q)$.

Case 1.a. Suppose that either $q \notin T$ or that $q$ belongs to the closure of $(X \cap Q)$. Then let $\Pi\left(Y^{*} \cap T\right)=\Lambda\left(Y^{*} \cap T\right)$ and let

$$
(\{q\} \cap T) \Pi(X \cap Q) \Pi\left(Y_{0} \cap T\right) \Pi(X \cap(T-Q)) \Pi\left(\left(Y^{*}-Y_{0}\right) \cap T\right) \Pi(\{p\} \cap T) .
$$

Case 1.b. Suppose that $q$ belongs to $T$ but not to the closure of $(X \cap Q)$. Here define $\Pi$ by interchanging $\Lambda$ and $\Lambda^{\prime}$ and $Q$ and $(T-Q)$ in the formulation of Case 1.a.

Case 2. If $q \in \bar{Y}^{*}$ define $\Pi\left(Y^{*} \cap T\right)=\Lambda\left(Y^{*} \cap T\right)$ and let

$$
(\{q\} \cap T) \Pi\left(Y^{*} \cap T\right)(\{p\} \cap T) .
$$

I.I. We will prove Lemma 4 by proving Lemmas 11 and 12 .

Lemma 11. If Condition 2 holds and $S \notin H$ but $S \in G$, then $T$ is totally orderable.

Proof. By definition, $S \in G^{\prime}$, so using the construction described in §I.G we define $Z, Y, Y^{*}, Y_{0}$, and $\Lambda$ for $K=S$. Since the endpoints of $S$ are in nontrivial components of $(S-T), Y$ is finite and $Y_{0}$ is the first term of $Y$. Hence we can define a total ordering $\Pi$ of $T$ having interval topology as follows. Let $\Pi\left(Y^{*} \cap T\right)=\Lambda\left(Y^{*} \cap T\right)$ and $\Pi\left(Q-Y^{*}\right)=\Delta\left(Q-Y^{*}\right)$ and $\Pi\left((T-Q)-Y^{*}\right)$ $=\Delta\left((T-Q)-Y^{*}\right)$. And let $\left(Q-Y^{*}\right) \Pi\left(Y_{0} \cap T\right) \Pi\left((T-Q)-Y^{*}\right) \Pi\left(\left(Y^{*}-Y_{0}\right) \cap T\right)$.

Lemma 12. If Condition 2 holds and $S \notin G$, then $T$ is totally orderable.

We will prove Lemma 12 by using Lemmas 8,9 , and 10 to help us reorder the points of $T$ in each term of $G$ and build a new underlying space $S_{0}$ from which 
by a simple sequence of complicated reorderings we can find a suitable ordering of the points of $T$.

First let us define replacement. Suppose that $I$ is a compact interval of $S$. Then we say that $\Phi(J)$ is a replacement of $I$ provided that:

(1) $J$ is a topological space and $\Phi$ is a total ordering of the points of $J$ having interval topology at every point of $J$,

(2) the subspace $(J \cap T)$ of $J$ is equivalent to the subspace $(I \cap T)$ of $I$, and

(3) $J$ is compact and connected, $\Phi(J)$ has the same first and same last points as $I$, and there are no trivial components of $(J-T)$.

We say that $\Phi(J)$ is a nice replacement of $I$ if $\Phi(J \cap T)$ orders $T$ nicely in $I$. Clearly, if $I$ is an interval of $S$ and $\Pi$ orders $T$ nicely in $I$, there is a nice replacement of $I$.

Now, assuming that Condition 2 holds, let us describe for each element $K$ of $G$ a replacement $\Phi\left(K_{0}\right)$ of $K$ and a collection $M_{K}$ of subintervals of $K_{0}$ as follows:

(1) If there is a nice replacement of $K$, let $\Phi\left(K_{0}\right)$ be a nice replacement of $K$ and let $M_{K}=0$.

(2) If precisely one endpoint of $K$ belongs to $P_{K}$ and $K \notin G^{\prime}$, let $\Phi\left(K_{0}\right)=\Delta(K)$ and let $M_{K}=\{K\}$.

(3) If $K \in G^{\prime}$ and $K=[p, q]$, where $p \in P_{K}$ and $q \notin P_{K}$, then let $\Phi\left(K_{0}\right)$ be a replacement of $K$ such that $K_{0}$ is the union of two subintervals $A$ and $B$, where $A$ is also a subinterval $[p, x]$ of $K$ such that $(p, x] \subset H$ and $B$ is a nice replacement of the subinterval $[x, q]$ of $K$. (Such exists by Lemma 8.) Let $M_{K}=\{A\}$.

(4) If $K=[p, q]$, where $p$ and $q$ are both in $P_{K}$, then let $\Phi\left(K_{0}\right)$ be a replacement of $K$ such that $K_{0}$ is the union of three subintervals $A, B$, and $C$, where $A$ and $C$ are subintervals $[p, x]$ and $[y, q]$ of $K$ such that $(p, x] \subset H$ and $[y, q) \subset H$ and $B$ is a nice replacement of the subinterval $[x, y]$ of $K$. (Such exists by Lemma 9.) Let $M_{K}=\{A, C\}$.

(5) If neither endpoint of $K$ belongs to $P_{K}$ and there is no nice replacement of $K$, let $\Phi\left(K_{0}\right)$ be a replacement of $K$ such that $\Phi\left(K_{0} \cap T\right)$ fails to have interval topology at at most finitely many points of $\left(K_{0} \cap T\right)$. Let $M_{K}=\left\{K_{0}\right\}$. To see that this possible, assume that neither endpoint of $K$ belongs to $P_{K}$. If $K \notin G^{\prime}$, then $\Delta(K \cap T)$ fails to have interval topology at at most finitely many points. But, if $K \in G^{\prime}$, we can construct $Z, Y, Y^{*}$, and $\Lambda$ as in $\S I$ I.G. Then $Y$ is finite and the endpoints of $Y^{*}$ are not in $T$ and $\Lambda\left(Y^{*} \cap T\right)$ has interval topology at every point of $\left(Y^{*} \cap T\right)$ and $\Delta\left(\left(K-Y^{*}\right) \cap T\right)$ fails to have interval topology at no more than finitely many points.

Let $\Phi(N)$ be the replacement of $S$ obtained by replacing each term $K$ of $G$ by $\Phi\left(K_{0}\right)$, and let $M$ denote the union of all $M_{K}$ for $K$ in $G$. The facts about $\Phi(N)$ and $M$ which we will wish to remember are given in Lemma 13.

Lemma 13. Suppose that Condition 2 holds and that $S \notin G$. Then there is a replacement $\Phi(N)$ of $S$ and a collection $M$ of compact disjoint intervals of $\Phi(N)$ having the following properties. 
(a) Every point at which $\Phi(T)$ fails to have interval topology belongs to some term of $M$.

(b) Define $H^{*}$ as the union of all open intervals of $\Phi(N)$ whose closures intersect $(T-Q)$ in a compact set disjoint from $\bar{Q}$. Then, if $I \in M$, there is an $e_{I} \in I$ such that $e_{I}$ is a limit point of $\left(N-\left(I \cup H^{*}\right)\right)$ and, if the set of all points of $(I \cap T)$ at which $\Phi(T)$ fails to have interval topology is infinite, they can be ordered in a simple sequence which is monotonic in $\Phi$ and whose sequential limit point is $e_{I}$.

(c) The set $M$ is countable and, if $i>0$ and $I \in M$, there is an open interval of $\Phi(N)$ containing $e_{I}$, no two points of which are in $(T-I)$ and are at a distance apart more than $i$ on the original line $R$.

Proof. Define $\Phi(N)$ and $M$ as above.

To see that the terms of $M$ are disjoint we use Lemma 10. Suppose that $I$ and $J$ are different elements of $M$ and that $p$ is a common point. There are terms $I^{\prime}$ and $J^{\prime}$ of $G$ such that $I \in M_{I^{\prime}}$ and $J \in M_{J^{\prime}}$, and, by the definition of $G$, either $\left(I^{\prime} \cap J^{\prime}\right)=\{p\}$ or $I^{\prime}=J^{\prime}$. But if $I^{\prime}=J^{\prime},(I \cap J)=0$ as in (4) above. But again by the definition of $G$, if $\left(I^{\prime} \cap J^{\prime}\right)=\{p\}$, one of $I^{\prime}$ and $J^{\prime}$, say $I^{\prime}$, does not intersect $H$ in a component having $p$ on its boundary. Then, by Lemma 10, the other endpoint of $I^{\prime}$ belongs to $P_{I^{\prime}}$. But, by our choice of $M_{I^{\prime}}$ in (2), (3), and (4) above, $I$ does not belong to $M_{I^{\prime}}$.

To prove (a), assume that $t \in T$. If $t$ is a point of some term $K$ of $G, \Phi\left(K_{0}\right)$ and $M_{K}$ were chosen in (1) through (5) above so that either $t$ belongs to some term of $M_{K}$ or $\Phi(T)$ has interval topology at $t$. And, if $t$ is not in some term of $G, \Phi(T)$ certainly has interval topology at $t$.

Now to see (b) let us assume that $K \in G$ and that $I \in M_{K}$. If there is a point $p \in\left(P_{K} \cap I\right)$, let $e_{I}=p$. Otherwise (see (1) and (5) above), $I$ is a replacement of $K$ and, since $S \notin G, K \neq S$, and $K$ has a boundary point which is an endpoint of $I$; let $e_{I}$ be such a point. (This is the only place in the proof of Lemma 12 where we use the fact that $S \notin G$.) When $e_{I}$ is so defined, by using Lemma 10 as in the preceding paragraph, we see that $e_{I}$ is in the closure of $(S-(K \cup H))$ and hence is also in the closure of $\left(N-\left(I \cup H^{*}\right)\right)$. And the number of points at which $\Phi(K)$ fails to have interval topology is infinite only if $e_{I} \in\left(P_{K} \cap I\right)$. So suppose that $e_{I} \in P_{K}$ and that $I=\left[e_{I}, x\right]$ and that $\left(e_{I}, x\right) \subset H$ and $x \notin P_{K}$. Let $V$ be the set of all points $v$ of $\left[e_{I}, x\right]$ such that either $v$ belongs to $(T-Q)$ or $v$ is between two points $t_{1}$ and $t_{2}$ of $(T-Q)$ in $\Delta$ and $\left(\left(t_{1}, t_{2}\right) \cap Q\right)=0$. Since $e_{I} \in P_{K}, V \neq 0$, and there is at least one point of $Q$ between each two components of $V$. in $\Delta$. So, since $x \notin P_{K}$ and $\left(e_{I}, x\right) \subset H, e_{I}$ is the sequential limit point of the components of $V$. This completes the proof of (b) since the points of $I$ at which $\Phi(T)$ fails to have interval topology are all endpoints of components of $V$.

Finally, (c) follows from the fact that we choose $\Delta$ to preserve the natural order 
of $T$ in the real line $R$, that (by (1) above) every term of $G$ which contains a term of $M$ intersects $T$, and that $\Phi$ is $\Delta$ except within single terms of $G$. And, if $I \in M_{K}$ and $K \in G$, the only term of $G$ which $e_{I}$ belongs to is $K$ and $e_{I}$ is an endpoint of $K$.

I.J. We are now ready to prove Lemma 12 and we will only need Lemma 13.

Proof of Lemma 12. Assume that Condition 2 holds and that $\Phi, N$, and $M$ have been defined satisfying Lemma 13 . We will now show by induction that $T$ is totally orderable.

Let $M_{1}, M_{2}, \cdots$ denote the terms of $M$.

Our induction hypothesis will be that we have a total ordering $\Delta_{n}$ of $T$ and a collection $N_{n}$ of compact intervals of $\Phi(N)$ having the following properties.

(1) The terms of $N_{n}$ do not intersect in $T$ and, if $n>0$, every term of $N_{n}$ is a subset of a term of $N_{n-1}$.

(2) If $n>0$ and $\Delta_{n}(T)$ fails to have interval topology at a point $t$, then $\Delta_{n-1}(T)$ fails to have interval topology at $t$ and $t \notin M_{n}$.

(3) If $\Delta_{n}(T)$ fails to have interval topology at a point $t$, then there is a term $I$ of $M$ containing $t$ and there is a term of $N_{n}$ which contains both $t$ and $e_{I}$ in its interior.

(4) If $X \in N_{n}$, then $\Delta_{n}(X \cap T)$ is either $\Phi(X \cap T)$ or $-\Phi(X \cap T)$ and no point of $(T-X)$ is between two points of $(X \cap T)$ in $\Delta_{n}$.

(5) If $n>0$ and $x \Delta_{n} y$ and $y \Delta_{n-1} x$, then $x$ and $y$ are in the same term of $N_{n-1}$ and, if $X$ is a term of $N_{n}$ containing either $x$ or $y$, no two points of $(X \cap T)$ are at a distance apart greater than $1 / n$.

(6) If $n>0$ and $x$ and $y$ belong to $T$ and no point is between $x$ and $y$ in $\Delta_{n-1}$, then no point is between $x$ and $y$ in $\Delta_{n}$.

First let us suppose that, for each positive integer $n$, we have defined $\Delta_{n}$ and $N_{n}$ satisfying the induction hypotheses, and we will now describe a total ordering $\Delta_{\infty}$ of $T$ which has interval topology. Suppose that $x$ and $y$ belong to $T$. If there is a smallest number $n$ such that $x$ and $y$ are not in the same term of $N_{n}$, let $x \Delta_{\infty} y$ if $x \Delta_{n} y$. Otherwise, for each $n$ there is a term $X_{n}$ of $N_{n}$ containing both $x$ and $y$. If there is an integer $m$ such that, for all $n>m, \Delta_{n}\left(X_{n} \cap T\right)=\Delta_{m}\left(X_{n} \cap T\right)$, then let $x \Delta_{\infty} y$ if $x \Delta_{m} y$; otherwise, let $x \Delta_{\infty} y$ if $x \Phi y$. By hypotheses (1) and (4) $\Delta_{\infty}$ is a total ordering of $T$.

To check that $\Delta_{\infty}(T)$ has interval topology let us assume that $\Delta_{\infty}(T)$ fails to have interval topology at a point $t$. By (3), if $\Delta_{0}(T)$ does not have interval topology at $t$, there is an $n$ such that $t$ belongs to $M_{n}$, and, by (2), then $\Delta_{n}(T)$ has interval topology at $t$. So there is an $n$ such that $\Delta_{n}(T)$ has interval topology at $t$. But then by (1), (4), (5), and (6), $\Delta_{\infty}(T)$ has interval topology at $t$.

So we only need to show that we can define $\Delta_{n}$ and $N_{n}$ as desired.

Let $\Delta_{0}=\Phi$ and $N_{0}=\{N\}$. These clearly satisfy our hypotheses.

Finally, suppose that, for some positive integer $k$, we have defined $\Delta_{n}$ and 
$N_{n}$ satisfying our induction hypotheses for all $n<k$; it remains to define $\Delta_{k}$ and $N_{k}$, also satisfying the hypotheses.

If there is no point of $\left(M_{k} \cap T\right)$ at which $\Delta_{k-1}(T)$ fails to have interval topology, let $\Delta_{k}=\Delta_{k-1}$ and let $N_{k}=N_{k-1}$; again our hypotheses are trivially satisfied.

So assume also that there is a point of $\left(M_{k} \cap T\right)$ at which $\Delta_{k-1}(T)$ fails to have interval topology. We will let e denote $e_{M_{k}}$ and let $X$ denote the term of $N_{k}$ containing $e$; by hypothesis (3), such exists and there is an open interval of $\Phi(N)$ containing $e$ and lying in $X$ which also contains every point of $\left(M_{k} \cap T\right)$ at which $\Delta_{k-1}(T)$ fails to have interval topology. Then by Lemma 13 (b) and (c), there is a $t$ belonging to $(T \cap X)$ such that $(e, t)$ is an open interval of $\Phi(N)$ which does not intersect $M_{k}$ and no two points of $(T \cap(e, t))$ are at a distance apart greater than $1 / k$ on the original real line $R$. Let $d$ denote the end point of $\left(M_{k} \cap X\right)$ different from $e$.

We will define for each of various cases a point $p_{0}$ of $(e, t)$, a disjoint collection $L$ of subintervals of $\left(e, p_{0}\right)$, and a total ordering $\Omega$ of $\left(\left[d, \rho_{0}\right] \cap T\right)$. We will let $L^{\prime}$ be the union of $L$ and the set of all components of $\left(X-\left(d, \rho_{0}\right)\right)$. Then in every case we take $N_{k}=\left(\left(N_{k-1}-\{X\}\right) \cup L^{\prime}\right)$. Also if $x$ and $y$ belong to $T$ and are not both in $\left[d, p_{0}\right]$ we say that $x \Delta_{k} y$ if $x \Delta_{k-1} y$; and we define $\Delta_{k}$ of $\left(\left[d, p_{0}\right] \cap T\right)$ to be $\Omega$ if $\Delta_{k-1}$ of $(X \cap T)$ is $\Phi$ and to be $-\Omega$ if $\Delta_{k-1}$ of $(X \cap T)$ is $-\Phi$. We are now assured that hypotheses (5) and (1) are satisfied; and by a simple check one will be able to see that the other hypotheses are satisfied by our construction of $p_{0}, L$, and $\Omega$.

Let $V^{\prime}$ be the set of all components of $(X-T)$ having precisely one endpoint in $([d, e] \cap T)$. If there is a term $v$ of $V^{\prime}$ and a point $x$ of $X$ and a point $y$ of $(T-X)$ and there is no point of $T$ between $x$ and $y$ in $\Delta_{k-1}(T)$ and $x$ is the only endpoint of $v$ in the interior of $X$, then let $V=\left(V^{\prime}-\{v\}\right)$; otherwise, let $V=V^{\prime}$. By Lemma 13(b), if $V$ is infinite, the terms of $V$ can be ordered in a simple sequence $v_{1}, v_{2}, \cdots$, which is monotonic in $\Phi$ and whose sequential limit point is $e$. If $V$ is finite, let $j$ denote the number of terms of $V$ and let $v_{1}, v_{2}, \cdots, v_{j}$ denote the terms of $V$ ordered monotonically in $\Phi$ so that $v_{1}$ is not between $v_{2}$ and $e$. For each $n$, let $d_{n}$ be a point of $v_{n}$ and let $b_{n}$ be the endpoint of $v_{n}$ which belongs to $T$.

In every case assume that $d \Phi e \Phi t$; otherwise, reverse all of the orders. Also define $\Omega([d, e] \cap T)=\Phi([d, e] \cap T)$.

Case 1. Suppose that $e$ belongs to $\bar{U}^{\prime}$, where $U^{\prime}$ is the union of all components of $((e, t)-T)$ having precisely one endpoint in $T$. Let $U$ denote the set of all such components and, for $u \in U$, let $b(u)$ denote the endpoint of $u$ belonging to $T$. If $u \in U, b(u)$ belongs to a term $I$ of $M$ and, as above, there is a term $u^{*}$ of $U$ such that $b\left(u^{*}\right)$ belongs to $I$ and, if $v$ is any other term of $U$ such that $b(v)$ belongs to $I, b\left(u^{*}\right)$ does not separate $v$ from $e_{I}$. Let $U^{*}$ be the set of all such $u^{*}$. Then let $u_{0}, u_{1}, \cdots$ be a sequence of terms of $U^{*}$ which is monotonic in $\Phi$ and has $e$ for its sequential limit point. Select $p_{n}$ belonging to $u_{n}$. We will assume 
without loss of generality that either, for all $n, b\left(u_{n}\right) \Phi p_{n}$ or, for all $n, p_{n} \Phi b\left(u_{n}\right)$. Let $L$ in Case 1 be the set of all $\left(p_{n}, p_{n+1}\right)$. We must complete our definition of $\Omega$ in Case 1 .

Case 1.a. Suppose that $V$ is finite and that $e \in \bar{U}^{\prime}$. Also suppose that $x \in\left(\left(p_{0}, d\right] \cap T\right)$ and that, for some $n>0, y \in\left(\left(p_{n}, p_{n-1}\right) \cap T\right)$. If $x \notin\left(p_{n}, p_{n-1}\right)$, define $y \Omega x$ if and only if $n \leqq j$ and $x \in\left(d_{n}, p_{n}\right)$ or if $n>j$ and $x \in\left(p_{n-1}, p_{j}\right)$. If $x \in\left(p_{n}, p_{n-1}\right)$ and $y \Phi x$, then define $y \Omega x$ if and only if either $n>j$ and $n-j$ is even or $n \leqq j$ and either $b_{n} \Phi d_{n}$ and $p_{n} \Phi b\left(u_{n}\right)$ or $d_{n} \Phi b_{n}$ and $b\left(u_{n}\right) \Phi p_{n}$.

Case 1.b. Suppose that $V$ is infinite and that $e \in \bar{U}^{\prime}$. And again suppose that $x \in\left(\left(p_{0}, d\right] \cap T\right)$ and that, for some $n>0, y \in\left(\left(p_{n}, p_{n-1}\right) \cap T\right)$. If $n$ is odd and $x \notin\left(p_{n}, p_{n-1}\right)$, then define $y \Omega x$ if and only if either $x \in\left(d_{n}, p_{n}\right)$ or $x \in\left(p_{m}, p_{m-1}\right)$, where $m$ is even. And if, for some integer $i, n=2 i-1$ and $x \in\left(p_{n}, p_{n-1}\right)$ and $y \Phi x$, then define $y \Omega x$ if and only if either $b_{i} \Phi d_{i}$ and $p_{n} \Phi b\left(u_{n}\right)$ or $d_{i} \Phi b_{i}$ and $b\left(u_{n}\right) \Phi p_{n}$. If $n$ is even and $x \notin\left(p_{n}, p_{n-1}\right)$, then define $y \Omega x$ if and only if $x \in\left(p_{m}, p_{m-1}\right)$, where $m$ is even and $m<n$. And, if $n$ is even and $x \in\left(p_{n}, p_{n-1}\right)$, define $y \Omega x$ if and only if either $y \Phi x$ and $n / 2$ is even or $x \Phi y$ and $n / 2$ is odd.

Case 2. Suppose that $e$ does not belong to the closure of $U^{\prime}$. By Lemma 13.b, $e$ is a limit point of $\left(N-\left(M_{k} \cup N^{*}\right)\right)$; hence there must be a point $t^{\prime}$ of $(e, t)$ such that $t^{\prime}$ belongs to $T$ and no component of $\left(\left(e, t^{\prime}\right)-T\right)$ has an endpoint belonging to $T$. Therefore we can select a sequence $p_{0}, p_{1}, \cdots$ converging monotonically to $e$ in $\Phi$ such that, for all $n \geqq 0, p_{n} \in\left(\left(e, t^{\prime}\right)-T\right)$ and, if $n>0$, between $p_{n}$ and $p_{n-1}$ in $\Phi$ there is a point $t_{n}$ of $(T-Q)$. By the definition of $Q$, the component of $T$ containing $t_{n}$ is trivia. and we can find sequences $r_{0}, r_{1}, \cdots$ and $s_{0}, s_{1}, \cdots$ of points of $(N-T)$ converging to $t_{n}$ monotonically in $\Phi$ such that $r_{0}=p_{n-1}$ and $s_{0}=p_{n}$. Then, in order to define $\Omega$ let us give a total ordering $\Phi^{\prime}$ of $\left(\left(p_{n}, p_{n-1}\right) \cap T\right)$ having interval topology and a last point but not first point. Let $\left.\Phi^{\prime}\left(\left(s_{0}, t_{n}\right) \cap T\right)=\Phi\left(\left(s_{0}, t_{n}\right)\right) \cap T\right)$ and let $\Phi^{\prime}\left(\left(t_{n}, r_{0}\right) \cap T\right)=-\Phi\left(\left(t_{n}, r_{0}\right) \cap T\right)$ and, for every positive integer $n$, let $\left(\left(s_{n-1}, s_{n}\right) \cap T\right) \Phi^{\prime}\left(\left(r_{n-1}, r_{n}\right) \cap T\right) \Phi^{\prime}\left(\left(s_{n}, s_{n+1}\right) \cap T\right) \Phi^{\prime}\left\{t_{n}\right\}$.

Now with $\Phi^{\prime}$ replacing $\Phi$ in $\left(\left(p_{n}, p_{n-1}\right) \cap T\right)$ we have exactly the same situation described in Case 1 where, for all $n, b\left(u_{n}\right) \Phi p_{n}$, and we can describe $\Omega$ as in this case. In Case 2, however, we let $L=0$ and $\left(\left[d, p_{0}\right) \cap T\right)$ has interval topology at every point.

When one checks the induction hypotheses, then, the proof of Lemma 12 and, hence, Lemma 4 and Theorem $I$ is complete.

II. Theorem II gives necessary and sufficient conditions for a subspace of any totally orderable space $R$ to be totally orderable. The conditions are more complicated than in the case where $R$ is the real line. The definition of $Q$ and Condition 2 must be expanded in case $T$ does not satisfy the first axiom of countability. And we need a new and difficult-to-state condition which will allow us to perform an induction analogous to that in the proof of Lemma 12 in \$I.J. However, we do not give the proof of Theorem II since it follows precisely the 
one given for the real line. The only really new, though not very difficult, element is that one must prove that Condition 3 is necessary. Otherwise, all of the lemmas of $\S I$ are true except that Condition 3 must be added to the hypotheses of Lemmas 4 and 12 and that we do not have (c) of Lemma 13. And the proofs of the lemmas are almost identical except that we must substitute Condition 3 for Lemma 13(c) and use a perhaps uncountable induction in the proof of Lemma 12.

One might mention that though we use $R$ and a particular ordering $\Omega$ of the points of $R$ in our definitions and conditions, in fact $Q$ and $H$ and the conditions are independent of the totally orderable space containing $T$.

II.A. For a given totally orderable topological space $R$ and a given subspace $T$ of $R$ make the following definitions.

(1) Let $\Omega$ be a total ordering of the points of $R$ such that the interval topology induced by $\Omega$ agrees with the given topology.

(2) If $p$ belongs to $T$, let $A(p)$ denote the set of all sequences of points of $T$ approaching $p$ which are monotonic in $\Omega$ and which have no subsequence of smaller cardinality approaching $p$.

(3) Closure here is taken in $R$. Let $Q$ denote the set of all points $q$ of $T$ such that: (a) if $p$ is the first or last point of $R$ in $\Omega$ or any point of $R$ not in the component of $T$ containing $q$, then the interval $[q, p]$ of $\Omega(R)$ does not intersect $T$ in a compact set, and (b) either the component of $T$ containing $q$ is not trivial or there are terms of $A(q)$ of different cardinality or there are terms $A$ and $A^{\prime}$ of $A(q)$ such that every subsequence of $A^{\prime}$ approaching $q$ has a limit point not in $\bar{A}$.

(4) Let $\Delta(S)$ be what might be called the Dedekind compactification of $\Omega(R)$. That is, let $p$ be a point of the space $S$ if either (a) $p$ is a point of $R$, or (b) $p$ is an initial interval of $\Omega(R)$ having no last point and such that no first point of $R$ follows this interval in $\Omega$. And let the topology of $S$ be the interval topology induced by $\Delta$ where, if $x$ and $y$ belong to $S$, then $x \Delta y$ if either (a) $x$ and $y$ belong to $R$ and $x \Omega y$ or (b) $x$ belongs to $R$ and $y$ to $(S-R)$ and some term of $y$ follows $x$ in $\Omega$, or (c) $x$ and $y$ are in $(S-R)$ and some term of $y$ follows every term of $x$ in $\Omega$, or (d) $y$ belongs to $R$ and $x$ to $(S-R)$ and some term of $x$ follows $y$ in $\Omega$.

(5) Closures here are taken in $S$. Let $H$ be the union of all open intervals $I$ of $\Delta(S)$ such that $(I \cap(T-Q))$ is compact and disjoint from $Q$. Let $H^{\prime}$ be the union of all open intervals of $\Delta(S)$ containing at most one point not in $H$, and let $G$ denote the collection of all intervals $[p, q]$ of $\Delta(S)$ such that $(p, q) \subset H^{\prime}$ but $p$ and $q$ are not in $H^{\prime}$.

(6) Finally, let $F$ denote the set of all terms $[p, q]$ of $G$, where $p \Delta q$ and there is no total ordering $\Gamma$ of $([p, q] \cap T)$ such that (a) $\Gamma([p, q] \cap T)$ has interval topology at every point, (b) if $p \in T, p$ is the first point of $\Gamma([p, q] \cap T)$ and, if $q \in T, q$ is the last point of $\Gamma([p, q] \cap T)$, and (c) if $p \notin T$, there is no first point of $\Gamma([p, q] \cap T)$, and, if $q \notin T$, there is no last point of $\Gamma([p, q] \cap T)$. 
The terms of $F$ can also be described in terms of various properties of the points of $[p, q]$ which are not in $H$.

II.B. THEOREM II. Suppose that $R$ is a totally orderable space and that $T$ is a subspace of $R$. Then, having made the definitions of §II.A, the following conditions are necessary and sufficient to insure that $T$ be a totally orderable space. Closures are taken in $T$.

Condition 1. If $(T-Q)$ is compact and does not intersect the closure of $Q$, then either $Q=0$ or $(T-Q)=0$.

Condition 2. If $I$ is an open interval of $\Omega(R)$ and $p \in(T-I)$ and $((I \cap(T-Q)) \cup\{p\})$ is compact and nontrivial and intersects the closure of $(I \cap Q)$ in $p$ and only in $p$, then (a) the component of $T$ containing $p$ is trivial, and (b) no term of $A(p)$ is uncountable.

Condition 3. If $S \notin G$, then, for each term I of $F$, there is a point $f(I) \in(T-I)$ such that, if $X \subset F$ and $X^{*}$ is the intersection of $T$ and the union of the terms of $X$ and $p \in\left(T-\left(X^{*} \cup f(X)\right)\right)$, then $p$ is in the closure of $X^{*}$ if and only if $p$ is in the closure of $f(X)$.

\section{BIBLIOGRAPHY}

1. I. L. Lynn, Linearly orderable subspaces of the real line, Abstract 579-18, Notices Amer. Math. Soc. 8 (1961), 161.

2. - Linearly orderable spaces, Proc. Amer. Math. Soc. 13 (1962), 454-456.

3. - Linearly orderable spaces, Abstract 599-48, Notices Amer. Math. Soc. 10 (1963), 261.

UNIVERSITY OF WISCONSIN, Madison, Wisconsin 